Published in final edited form as:

Bioconjug Chem. 2020 September 16; 31(9): 2211-2219. doi:10.1021/acs.bioconjchem.0c00384.

\title{
Electrostatic Conjugation of Nanoparticle Surfaces with Functional Peptide Motifs
}

\author{
Natalie Boehnke, \\ Koch Institute for Integrative Cancer Research, Massachusetts Institute of Technology, \\ Cambridge, Massachusetts 02139, United States \\ Kate J. Dolph, \\ Departments of Chemistry and Biology, Wellesley College, Wellesley, Massachusetts 02481, \\ United States
}

\author{
Valeria M. Juarez, \\ Department of Biomedical Engineering, University of Texas at San Antonio, San Antonio, Texas \\ 78249, United States
}

Julia M. Lanoha, Department of Bioengineering, Santa Clara University, Santa Clara, California 95053, United States

\section{Paula T. Hammond}

Koch Institute for Integrative Cancer Research and Department of Chemical Engineering, Massachusetts Institute of Technology, Cambridge, Massachusetts 02139, United States

\section{Abstract}

\begin{abstract}
We report the surface functionalization of anionic layer by layer nanoparticles (LbL NPs) with cationic tumor-penetrating peptides (TPPs) via electrostatic adsorption while retaining particle stability and charge characteristics. This strategy eliminates the need for structural modifications of the peptide and enables facile functionalization of surface chemistries difficult to modify or inaccessible via covalent conjugation strategies. We show that both carboxylated and sulfated LbL NPs are able to accommodate linear and cyclic TPPs and used fluorescence-based detection assays to quantify peptide loading per NP. We also demonstrate that TPP activity is retained upon adsorption, implying sufficient numbers of peptides take on the appropriate surface orientation, enabling efficient uptake of functionalized NPs in vitro, as characterized via flow cytometry and
\end{abstract}

\footnotetext{
Corresponding Author Paula T. Hammond -Koch Institute for Integrative Cancer Research and Department of Chemical Engineering, Massachusetts Institute of Technology, Cambridge, Massachusetts 02139, United States; hammond@mit.edu. Author Contributions

N.B. and P.T.H. designed the experiments and analyzed the data. N.B., K.J.D., and P.T.H. wrote the manuscript. N.B., K.J.D., V.M.J., and J.M.L. conducted experiments. All authors contributed to interpretation of the results and preparation of the paper.

The authors declare no competing financial interest.

ASSOCIATED CONTENT

Supporting Information

The Supporting Information is available free of charge at https://pubs.acs.org/doi/10.1021/acs.bioconjchem.0c00384.

Detailed materials and methods, supporting figures, and references (PDF)

Complete contact information is available at: https://pubs.acs.org/10.1021/acs.bioconjchem.0c00384
} 
deconvolution microscopy. Overall, we believe that this strategy will serve as a broadly applicable approach to impart electrostatically assembled NPs with bioactive peptide motifs.

\section{Graphical Abstract}

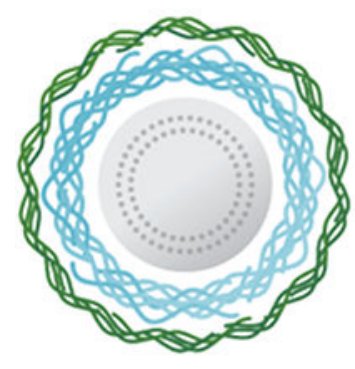

anionic LbL NP
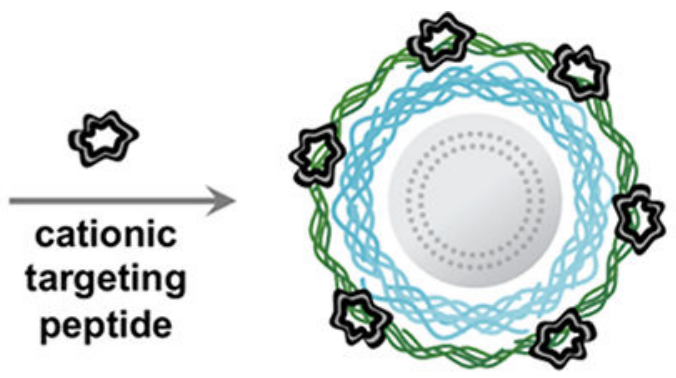

electrostatically conjugated anionic NP

- Reagent-free, charge-based conjugation strategy

- Retention of particle charge and stability

- Peptide adsorption stable to physiologic conditions

- Bioactivity of peptides maintained

\section{INTRODUCTION}

Nanoparticles (NPs) are commonly used for targeted drug delivery applications due to their tunable properties, including the ability to functionalize particle surfaces with specific targeting motifs to enhance delivery to a specific tissue type or site of disease. This is most commonly achieved through the covalent addition of tumor-penetrating peptides (TPPs). ${ }^{1-3}$ To install such tumor-targeting capabilities onto NP surfaces, chemical modifications of both the targeting motif and the particle are required, increasing synthetic complexity and cost as well as limiting the types of NPs amenable to this approach. Alternatively, NPs can be noncovalently functionalized with peptides through hydrophobic or gold-thiol interactions. ${ }^{4,5}$ However, these approaches again require the chemical modification of the targeting motifs and are limited to select types of NP formulations. Therefore, it would be advantageous to consider NP synthesis and functionalization methods that are not limited by such chemical modifications and are compatible with a broad range of nanomaterials. Furthermore, it would be particularly interesting to adapt such a method to an already existent NP modification platform.

NPs synthesized via the layer-by-layer (LbL) method of self-assembly comprise a powerful and modular platform to develop multifunctional and targeted nanomedicines. ${ }^{6,7} \mathrm{LbL}$ assembly consists of iterative charge-based adsorption of functional polyelectrolytes onto colloidal substrates to incorporate polymers of varying chemical complexity, including polysaccharides and polypeptides, without the use of multistep conjugation chemistries. A typical LbL construct consists of a charged NP core, such as a drug-loaded liposome, 
polycation layers for enhanced endosomal escape, and a polyanionic outer layer that presents a densely charged and hydrated surface required for successful systemic delivery, immune evasion, and extended plasma half-life. ${ }^{6,7}$ Additionally, therapeutic biomaterials can be readily incorporated within the electrostatic film construct, including nucleic acids such as siRNA and proteins, for effective and staged combination therapy delivery. ${ }^{8-10}$ Tumortargeting capabilities can be incorporated through the addition of specific outer layers that provide a negatively charged "stealth" surface with known receptor interactions, such as hyaluronic acid for CD44 targeting. ${ }^{11-14}$ This has enabled the successful development of powerful, targeted combination therapy delivery vehicles with proven efficacy in numerous aggressive and recurrent tumor models. ${ }^{8,10,13,14}$ Moreover, we have recently shown that LbL assembly can be used to easily and rapidly generate NP libraries with varying polyanionic outer layers, enabling the discovery of new surface chemistries with high affinity for ovarian cancer cells. ${ }^{15}$

Inspired by the ease with which film components of LbL NPs can be interchanged to create nanocarriers with new and distinct properties, we posed the question of whether electrostatic interactions can also be used to replace covalent NP functionalization. We sought to establish that electrostatic adsorption alone could serve as a means of introducing a functional peptide on the surface of the NP while (a) introducing the active functionality of the peptide and (b) retaining the net negative charge and hydrophilic properties that enable longer plasma half-lives, decreased nonspecific cellular interactions, and lowered clearance. Specifically, we utilized charge-based electrostatic interactions to conjugate cationic TPP motifs onto anionic LbL NP surfaces.

TPPs comprise a class of targeting peptides including iRGD, LyP-1, and LinTT1. ${ }^{16}$ TPPs improve NP accumulation in tumor tissue through an endocytic transport pathway initiated by the binding of the TPP to a tumor specific primary receptor, such as integrins ${ }^{17}$ (iRGD) and $\mathrm{p} 32^{18,19}$ (LyP-1, LinTT1). This is followed by peptide cleavage and internalization by the second receptor, neuropilin-1 (NRP-1), common for all TPPs bearing CendR motifs. ${ }^{3,20}$ Because the efficacy of TPPs to enhance cargo delivery has been well explored in ovarian cancer, where overexpression of both integrins and p32 has been characterized, ${ }^{21}$ we chose to demonstrate the efficacy of our conjugation approach in the context of high grade serous ovarian cancer (HGSOC), which is one of the most lethal gynecological malignancies. Further, numerous therapeutic challenges are associated with ovarian cancer, including metastasis to other organs in the intraperitoneal space and the need to address resistance with combination therapies that induce higher systemic toxicities, thereby providing an area with urgent need for the development of targeted therapies; these issues could be addressed by selective nanoparticle delivery strategies. ${ }^{22}$ Using a liposomal LbL NP library containing polypeptide and polysaccharide outer layers bearing either carboxylate or sulfate groups, we demonstrate the adsorption of two cationic TPPs with retention of NP stability and anionic properties as well as peptide activity.

\section{RESULTS AND DISCUSSION}

The LbL NP library was prepared using an anionic liposomal core, chosen for its translational relevance, drug loading potential, and tunable surface charge, as well as 
uniform and controlled size. Specifically, we elected to formulate liposomes composed of 61.7 mol \% 1,2-distearoyl-sn-glycero-3-phospho-(1'-rac-glycerol) (DSPG), $33.3 \mathrm{~mol} \%$ cholesterol, and $5 \mathrm{~mol} \%$ 1,2-dioleoyl-sn-glycero-3-phosphoethanolamine (DOPE) due to the formulation's clinical applicability and its prior use in colloidal LbL. ${ }^{23,24}$ DOPE was incorporated to label the liposomes with fluorescent tags for downstream analysis. Poly-Larginine (PLR) was chosen as the polycation due to its established endosomal escape and strong electrolyte properties. ${ }^{9}$ The outer layers selected for our library screen included three carboxylated polyelectrolytes: hyaluronate (HA), poly-l-aspartate (PLD), poly-l-glutamate (PLE), and one sulfated polyelectrolyte: dextran sulfate (DXS). HA and DXS are classified as natural polysaccharides whereas PLD and PLE are synthetic homopolypeptides. Dynamic light scattering (DLS) was utilized to monitor the size, dispersity, and zeta potential $(\zeta)$ of the NPs throughout the synthesis and purification process. ${ }^{24,25}$

We chose to adsorb two different TPPs onto the surfaces of our LbL NP library, LyP-1 and LinTT1 (Figure 1A), both bearing a net charge of +3 with similar molecular weights of 992.2 and 920.0 Da, respectively. We employed preliminary DLS characterization to monitor NP size and $\zeta$ before and after TPP adsorption to determine whether these peptides can be adsorbed while maintaining NP stability and preventing loss or inversion of surface charge. We note that LyP-1 is a cyclic disulfide (Figure 1B), whereas LinTT1 is a linear peptide (Figure 1C), enabling comparisons of adsorption effects based on architectural differences. The objective of the adsorption step was to introduce enough of the functional peptides onto the surface to achieve effective ligand functionalization, while maintaining a net negative charge sufficient to maintain stability of the LbL NP. While $\zeta$ measurements alone do not confirm NP interactions with cationic LyP-1, we believe it to be a useful tool for preliminary screening of these potential charge-charge interactions, similar to how we employ DLS characterization to monitor colloidal LbL assembly. ${ }^{24}$

A range of weight equivalents (wt equiv, with respect to liposome core) of LyP-1 was titrated onto the surface of LbL NPs in 1 mM HEPES, and DLS was utilized to monitor NP size and $\zeta$ in response to addition of the cationic peptide (Figure 2A). PLD, PLE, and DXS NPs shifted from around $-50 \mathrm{mV}$ without LyP-1 to $-25 \mathrm{mV}$ at 1 wt equiv of LyP-1, and HA NPs shifted from $-31.1 \pm 3.1$ to $-21.9 \pm 0.6 \mathrm{mV}$. This dose-dependent decrease in NP $\zeta$ points to potential electrostatic compensation between the anionic nanoparticles and cationic LyP-1 upon peptide adsorption. Additionally, particle diameter did not change drastically, indicating that NPs remained stable to LyP-1 addition at the tested concentrations. The rise in $\zeta$ above $-30 \mathrm{mV}$, which took place at weight equivalents higher than 1 , started to induce NP instability, crossing a threshold for colloidal stability, ${ }^{24,26}$ as the positive charge of the peptide reduces the original negative surface charge of the NP. We note that HA NPs were initially less charged than the other LbL NPs, though stable, which can potentially be explained by the lower charge density of HA and heavy hydration of the polysaccharide. However, because we did not observe NP aggregation as the HA NPs decreased below -30 $\mathrm{mV}$ at LyP-1 equiv from 0.125 to 1 based on the constant NP size measured with DLS, we concluded that particle stability was retained.

Because LyP-1 is much smaller and of lower charge density than polyelectrolytes typically utilized for electrostatic LbL assembly, we initially assessed the stability of the peptide-NP 
interaction to purification conditions (Figure 2B). NPs were mixed with $0.25 \mathrm{wt}$ equiv of LyP-1 in $1 \mathrm{mM}$ HEPES and purified via tangential flow filtration (TFF) to remove unbound peptide from solution. ${ }^{25}$ DLS was used to track the $\zeta$ of NPs before LyP-1 adsorption, and after adsorption pre- and postpurification. We hypothesized that the decrease in $\zeta$ observed after peptide adsorption should be retained after purification if the LyP-1-NP interactions are stable to TFF conditions. Indeed, the results support our hypothesis, indicating that LyP-1 remained bound to the LbL NPs. For PLD, PLE, and DXS NPs, $\zeta$ values became more positive after purification. Because $\zeta$ measurements are sensitive to fluctuations in solution conditions, such as salt and buffer concentration, we believe this observed increase in $\zeta$ reflects the removal of counterions and adsorption buffer (1 mM HEPES) during the purification process.

To extend this approach, we titrated LbL NPs with LinTT1 and found similar dosedependent effects on particle $\zeta$ with retention of particle stability until the measured $\zeta$

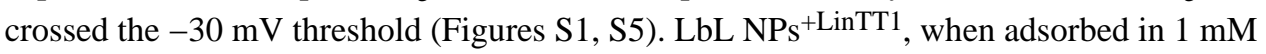
HEPES, were found to be similarly stable to TFF conditions (Figure 2C). PLE NPs without peptide exhibited $\zeta$ of $-52.7 \pm 3.0 \mathrm{mV}$ whereas the $\zeta$ of PLE NP ${ }^{+\mathrm{LyP}-1}$ and PLE NP ${ }^{+\mathrm{LinTT} 1}$ were $-28.8 \pm 0.5$ and $-29.9 \pm 2.5 \mathrm{mV}$, respectively.

Peptide loading of the LbL NP library postpurification was assessed using a fluorometric assay. Because Lyp-1 is a cyclic disulfide, we chose to use a probe that could quantify resulting thiol groups following reduction of LyP-1 with tris(2-carboxyethyl)phosphine (TCEP) (Figure 3A). ${ }^{27}$ Using this probe, ABD-F, we determined the \% peptide retention corresponded to high peptide loading, ranging from 3700 to 8400 LyP-1 molecules per NP. Specifically, PLD NPs retained 53.5 $\pm 0.3 \%$, PLE NPs retained $48.6 \pm 0.9 \%$, HA NPs retained $80.5 \pm 1.0 \%$, and DXS NPs retained $110 \pm 2.5 \%$ of the 0.2 wt equiv of LyP-1 (Figure 3B). Interestingly, two trends emerged from these results. The first was that DXS NPs retained much higher amounts of LyP-1 than the tested carboxylated LbL NPs. We attribute this to the stronger binding of the sulfate groups with the cations present in LyP-1, as was also reported previously ${ }^{28,29}$ and which may be a result of differences in base ion polarizability. The second trend showed that the two polysaccharide-coated NPs retained higher amounts of LyP-1 than the polypeptide-coated NPs. This could be due to increased hydrogen bonding capabilities of the sugar backbones with the peptide ligands compared to the polypeptides. To quantify LinTT1 loading via this assay, LinTT1 with a free cysteine at the $\mathrm{N}$ terminus (LinTT1-SH) was synthesized. ${ }^{30}$ We found that peptide loading of LyP-1 and LinTT1-SH was similar (Figure S2).

For subsequent experiments, we chose to narrow our focus to PLE and DXS NPs. This was done to compare polypeptide and polysaccharide as well as carboxylate and sulfate chemistries in a biologically relevant context. We have previously established that PLE NPs have a high affinity for ovarian cancer cells but are extracellularly associated, and we have also shown that DXS NPs are poorly taken up by ovarian cancer cells. ${ }^{15}$ Therefore, we hypothesized that the adsorption of LyP-1 or LinTT1 should be observable by either an increase in NP-cell association or change in intracellular trafficking of the NPs. 
As solution conditions are critical for colloidal LbL stability and retention of cargo, we compared the adsorption of LyP-1 onto LbL NPs in water, dilute HEPES (1 mM, pH 7.4. for slight $\mathrm{pH}$ buffering), and $25 \mathrm{mM}$ HEPES $+20 \mathrm{mM} \mathrm{NaCl}$ (pH 7.4, for $\mathrm{pH}$ control and charge shielding) ${ }^{24}$ (Figures 2A, S3A). We found that 0.5 wt equiv of LyP-1 was stably incorporated onto the LbL NP surfaces in both the HEPES and the HEPES + NaCl solution conditions, indicating that the presence of slight buffering conditions improves LyP-1 adsorption. Conversely, adsorption of LyP-1 in water resulted in particle aggregation at this concentration (Figure S3A). This is in agreement with previous studies where we have shown that addition of HEPES and dilute salt can lead to more stable LbL NPs capable of retaining higher amounts of nucleic acid cargo. ${ }^{24}$ However, at a lower wt equiv of LyP-1, all solution conditions resulted in stable NPs with no difference in particle functionality (Figure $\mathrm{S} 3 \mathrm{~B}, \mathrm{C})$.

We further evaluated the stability of peptide interactions with the NP surface to increasing salt $(\mathrm{NaCl})$ concentrations. For colloidal LbL NPs, layers are built up primarily through charge-charge interactions in combination with secondary interactions. The same premise applies for TPP adsorption onto the NP surface, though these interactions are fewer because of the peptides' lower net charge compared to polyelectrolytes. Therefore, we hypothesized that if the peptides were only weakly or transiently associated with the NP surface, dilute amounts of $\mathrm{NaCl}$ would disrupt these noncovalent interactions through excess shielding, detectable via a change in NP diameter or $\zeta$. We first established the stability of PLE and DXS NPs to $\mathrm{NaCl}$ and found that they were stable to 50 and $100 \mathrm{mM} \mathrm{NaCl}$, respectively (Figure 4, Figure S4). At and above these $\mathrm{NaCl}$ concentrations, loss of colloidal stability was observed either through particle aggregation (indicated by a significant increase in measured particle size in DLS) or reduction of $\zeta$. We chose $200 \mathrm{~nm}$ as the threshold for stable NP diameter based on prior studies. ${ }^{24}$ Addition of 0.5 wt equiv of LyP-1 onto these NPs did not change the colloidal stability from what was observed for the NPs without LyP-1 (Figure 4A). Moreover, $\zeta$ measurements for PLE NP ${ }^{+\mathrm{LyP}-1}$ and DXS NP ${ }^{+\mathrm{LyP}-1}$ read consistently less negative than PLE NP and DXS NP counterparts up to the point of general particle destabilization, indicating that the LyP-1 NP interactions are as stable as the electrostatically assembled LbL NPs (Figure S4). We elected to test a higher concentration of TPPs for these experiments to ensure that relatively high amounts of peptide did not have an adverse effect on NP physical properties.

We were surprised to find that PLE NP ${ }^{+\mathrm{LinTT} 1-\mathrm{SH}}$ and DXS NP ${ }^{+\mathrm{LinTT} 1-\mathrm{SH}}$ destabilized at lower salt concentrations of 5 and $50 \mathrm{mM} \mathrm{NaCl}$, respectively, pointing to the presence of LinTT1 having a disruptive effect on NP stability (Figure 4B). Because the two TPPs exhibit the same overall net charge $(+3)$ as well as similar molecular weights and polar surface areas, we hypothesized that structural differences, specifically cyclic vs linear, between the two peptides may explain the observed differences. Moreover, stability of cyclic peptides for nanoparticle surface modification over linear peptides has been reported in other NP systems., ${ }^{8}$ It is also well established that macrocyclic peptides are conformationally constrained and retain more rigid structures compared to linear peptides, accounting for improved properties such as proteolytic stability. ${ }^{4,31}$ To decouple the effects of linear peptide structure and charge and to rule out that the presence of a linear peptide by itself could contribute to NP destabilization, we synthesized two LinTT1 analogues with decreasing 
charge, wherein Lys and Arg residues were replaced with Gly (Figure 4C). These analogues were adsorbed onto PLE and DXS NPs, and NP stability in a $\mathrm{NaCl}$ solution was again assessed (Figure S5, Figure 4D). It was noted that PLE NP and PLE NP+LinTT1+1 Charge exhibited the same response to increasing salt concentration, with significant aggregation observed only at $100 \mathrm{mM} \mathrm{NaCl}$, though a slight increase in particle diameter was observed at $50 \mathrm{mM} \mathrm{NaCl}$, consistent with the observations in Figure 4B. However, PLE NP +LinTT1+2 Charge exhibited much more significant aggregation at $100 \mathrm{mM} \mathrm{NaCl}$, potentially indicating a shift in NP stability. PLE NP+LinTT1+3 Charge exhibited much more drastic aggregation at both 50 and $100 \mathrm{mM} \mathrm{NaCl}$, indicating that the higher charge on the linear peptide has a destabilizing effect on PLE NPs. The same trend was observed for the DXS NPs, where significant aggregation at $50 \mathrm{mM} \mathrm{NaCl}$ was only observed for DXS NP +LinTT1+3 Charge (Figure 4D). Ultimately, these data indicate that the presence of LinTT1 analogues without high cationic charge does not destabilize LbL NPs and that both high cationic charge and linear peptide structure are required for destabilization. This is in contrast to the lack of destabilizing effects of LyP-1. Potentially, the linear structure of LinTT1 may enable it to act as a more flexible polycation than LyP-1, and it therefore may form a strong polyion complex with the outer layer of the NP more effectively and thus better shield charge. The cyclic nature of LyP-1 may restrict it from covering the surface as effectively.

We additionally incubated PLE and DXS NPs with and without adsorbed TPPs ( $0.2 \mathrm{wt}$ equiv) in Roswell Park Memorial Institute (RPMI) 1640 cell culture medium supplemented with $10 \%$ fetal bovine serum (FBS) to evaluate colloidal stability of TPP-bearing NPs (Figure S6). We observed that PLE and DXS NPs retained stable and uniform sizes below $200 \mathrm{~nm}$, and adsorption of $0.2 \mathrm{wt}$ equiv of LyP-1 did not impact serum stability. Because RPMI 1640 contains $150 \mathrm{mM} \mathrm{NaCl}$ along with several additional salt components, we hypothesized that the amino acids and serum proteins present in the media may provide stabilizing effects of the tested colloidal NPs. Consistent with our prior observation, LinTT1 adsorption had a more destabilizing effect. Both PLE NP ${ }^{+L i n T T 1}$ and DXS NP ${ }^{+L i n T T 1}$ increased in size in response to incubation in the serum-containing media, indicating a potential loss of colloidal stability. Overall, our findings underscore the need to fully characterize the adsorption of any new peptide motifs on LbL NPs to account for the effects of charge and conformation.

Having confirmed the range of stability of the peptide-NP interactions, we evaluated whether these TPPs retain their function when adsorbed onto a NP surface. To this end, LyP-1 and LinTT1 were separately adsorbed onto fluorescently labeled PLE and DXS NPs, purified, and incubated with high grade serous ovarian cancer cells (OVCAR8) for $24 \mathrm{~h}$ using water as the solution condition. Subsequent NP-cell association was analyzed via flow cytometry (Figure 5). As LyP-1 and LinTT1 have both been established to target p32, overexpressed on the surface of cancer cells, including the OVCAR8 cell line,${ }^{21}$ the addition of tumor-penetrating peptides should be observable by either an increase in NP-cell association or a change in NP uptake and intracellular trafficking, the latter being not detectable through flow cytometry. We found that PLE NP ${ }^{+L y P-1}$ had a slight, though not statistically significant, improvement in NP-cell association compared to PLE NP while PLE NP ${ }^{+L i n T T 1}$ resulted in a more noticeable increase in NP-cell association (Figure 5B). 
We hypothesized that we did not observe a more drastic difference because there may not be much room for improvement in the high affinity PLE NP-cell association, as evidenced by greater than $96 \%$ of all analyzed cells exhibiting higher fluorescence $\left(\mathrm{NP}^{+}\right.$population) than the untreated control. Instead, the peptide modification may result in a change in PLE NP intracellular trafficking. We were initially surprised to find that DXS NPs did not have higher NP-cell association when modified with either LyP-1 or LinTT1 (Figure 5B). However, because sulfate (NP)-cation (TPP) interactions are stronger than carboxylate (NP)-cation (TPP) interactions and we characterized higher stability of DXS NPs to salt compared to PLE NPs, it may be that the peptide-DXS interactions are too strong to allow for peptide-receptor interactions, as the cationic residues, which are responsible for receptor binding, are strongly associated with the sulfate groups (Figure 4A,B). ${ }^{32}$ This experiment was repeated using PLE and DXS NPs wherein the TPPs were adsorbed using $25 \mathrm{mM}$ HEPES + $20 \mathrm{mM} \mathrm{NaCl}$ as the solution conditions to evaluate if addition of salt or buffering conditions results in a more discernible change in NP-cell association, perhaps through changing the orientation of the adsorbed peptides on the NP surface to be more accessible by cell surface receptors. However, no differences were observed via flow cytometry (Figure $\mathrm{S} 3)$. Moreover, the above results were also observed in a second ovarian cancer cell line, COV362 (Figure S7).

As the toxicity of cationic materials, including peptides, has been reported, we carried out cytotoxicity studies using the OVCAR8 cell line to evaluate the biocompatibility of LbL NPs adsorbed with LyP-1 and LinTT1 (Figure S8). ${ }^{33}$ As expected, the tested LbL NPs exhibited good biocompatibility, and no change in viability was observed after the addition of TPP. This is likely due to the anionic nature of the NP being preserved, mitigating the effects of the cationic peptides.

To evaluate the effects of TPP adsorption on intracellular trafficking of PLE NPs, we carried out deconvolution microscopy (Figure 6). OVCAR8 cells were dosed with fluorescently labeled NPs for $24 \mathrm{~h}$ prior to fixing, staining, and imaging. PLE NPs were extracellularly associated on the OVCAR8 cell membranes with little to no internalization observed, consistent with our previously published findings (Figure 6A) ${ }^{15} \mathrm{PLE} \mathrm{NP}^{+\mathrm{LyP}-1}$ on the other hand was efficiently internalized, colocalizing with intracellular vesicle membranes, though some of the NPs were still retained on the cell surface (Figure 6B). This indicates that function of LyP-1 is retained when adsorbed onto the NP surface in addition to the NP surface chemistry characteristics. To rule out that the observed NP internalization was not simply due to modulating the charge of the NP surface through addition of a cationic material, we generated NPs layered with a propargyl-modified PLE (pPLE) NP for covalent modification with the neutral TPP, iRGD. ${ }^{23}$ While the initial receptor of iRGD differs from LyP-1, both are ultimately internalized via neuropilin-1 (NRP-1), and we therefore conjectured that we would see similar trafficking patterns for the iRGD and LyP-1 modified NPs if uptake differences were a result of NRP-1-mediated internalization. ${ }^{3}$ Indeed, this is what we observed (Figure 6C). PLE NP ${ }^{+L i n T T 1}$ were also internalized, though to a lesser extent than PLE ${ }^{+\mathrm{Ly}-1}$ (Figure 6D). We also noted large patches of NP on the cell surface and attribute this to the potential self-aggregating effect of LinTT1 on LbL NPs that we had seen previously at physiological salt conditions (Figure 4). As an additional control, we synthesized a scrambled version of LinTT1, keeping the charge the same at +3 . When 
adsorbed onto PLE NPs and incubated with OVCAR8 cells, we observed the same clusters of NPs on the cell surface as seen with PLE NP ${ }^{+L i n T T 1}$ but without particle internalization (Figure 6E). This again indicated that the observed NP internalization was due to the retention of TPP function when adsorbed onto PLE NP surfaces.

\title{
CONCLUSIONS
}

We demonstrated that electrostatic adsorption is a new approach to surface conjugation of LbL NPs with charged, functional peptides. We showed the stability of the surface-adsorbed TPPs to purification conditions and salt with retention of their bioactivity in vitro. This bioactivity was demonstrated in a change in the intracellular trafficking of the LbL NPs, which led to greatly increased internalization of the NPs compared to unfunctionalized LbL NPs. Ultimately, this approach could be broadly applicable to the functionalization of a large number of drug delivery systems, particularly in the context of the blood-brain barrier, wherein numerous cationic shuttle peptides are being employed to enhance nanoparticle penetration into brain tissue.

\section{Supplementary Material}

Refer to Web version on PubMed Central for supplementary material.

\section{ACKNOWLEDGMENTS}

\begin{abstract}
The authors thank the MIT Koch Institute Swanson Biotechnology Center, which is supported by the Koch Institute Core Grant P30-CA14051 from the NCI. Specifically, we thank Glenn Paradis in the Flow Cytometry Facility and Jeffrey Kuhn in the Microscopy Core. We thank Joelle Straehla, Tamara Gomez Dacoba, Elad Deiss-Yehiely, and Santiago Correa for helpful discussion and advice.

Funding

This work was supported by grants from the Koch Institute's Marble Center for Cancer Nanomedicine as well as the Bridge Project, a partnership between the Koch Institute for Integrative Cancer Research at MIT and the DanaFarber/Harvard Cancer Center. Resources were provided in part by the Koch Institute Support Grant (P30CA14051) from the National Cancer Institute. N.B. gratefully acknowledges funding support from the Koch Institute's Marble Center for Cancer Nanomedicine for a postdoctoral fellowship as well as from the Department of Defense Peer Reviewed Cancer Research Program for a Horizon Award (W81XWH-19-1-0257). K.J.D. was supported by a grant from the Wellesley College Susan Todd Horton 1910 Internship Fund. V.M.J. was supported by the Massachusetts Institute of Technology Summer Research Program (MSRP) and NIGMS MARC U*STAR GM007717.
\end{abstract}

\section{REFERENCES}

(1). Rosenblum D, Joshi N, Tao W, Karp JM, and Peer D (2018) Progress and challenges towards targeted delivery of cancer therapeutics. Nat. Commun 9, 1-12. [PubMed: 29317637]

(2). Shi JJ, Kantoff PW, Wooster R, and Farokhzad OC (2017) Cancer nanomedicine: progress, challenges and opportunities. Nat. Rev. Cancer 17, 20-37. [PubMed: 27834398]

(3). Ruoslahti E (2017) Tumor penetrating peptides for improved drug delivery. Adv. Drug Delivery Rev 110, 3-12.

(4). Choi SJ, Jeong WJ, Kang SK, Lee M, Kim E, Ryu DY, and Lim YB (2012) Differential SelfAssembly Behaviors of Cyclic and Linear Peptides. Biomacromolecules 13, 1991-1995. [PubMed: 22676488]

(5). Sardan M, Yildirim A, Mumcuoglu D, Tekinay AB, and Guler MO (2014) Noncovalent functionalization of mesoporous silica nanoparticles with amphiphilic peptides. J. Mater. Chem. B 2, 2168-2174. [PubMed: 32261500] 
(6). Correa S, Dreaden EC, Gu L, and Hammond PT (2016) Engineering nanolayered particles for modular drug delivery. J. Controlled Release 240, 364-386.

(7). Alkekhia A, Hammond PT, and Shukla A Layer-by-Layer Biomaterials for Drug Delivery. Annu. Rev. Biomed. Eng 2020, 22, 1 [PubMed: 32084319]

(8). Gu L, Deng ZJ, Roy S, and Hammond PT (2017) A Combination RNAi-Chemotherapy Layer-byLayer Nanoparticle for Systemic Targeting of KRAS/P53 with Cisplatin to Treat Non-Small Cell Lung Cancer. Clin. Cancer Res 23, 7312-7323. [PubMed: 28912139]

(9). Deng ZJ, Morton SW, Ben-Akiva E, Dreaden EC, Shopsowitz KE, and Hammond PT (2013) Layer-by-Layer Nanoparticles for Systemic Codelivery of an Anticancer Drug and siRNA for Potential Triple-Negative Breast Cancer Treatment. ACS Nano 7, 9571-9584. [PubMed: 24144228]

(10). Choi KY, Correa S, Min J, Li JH, Roy S, Laccetti KH, Dreaden E, Kong S, Heo R, Roh YH, Lawson EC, Palmer PA, and Hammond PT Binary Targeting of siRNA to Hematologic Cancer Cells In Vivo Using Layer-by-Layer Nanoparticles. Adv. Funct. Mater 2019, 29, 1900018 [PubMed: 31839764]

(11). Aruffo A, Stamenkovic I, Melnick M, Underhill CB, and Seed B (1990) Cd44 Is the Principal Cell-Surface Receptor for Hyaluronate. Cell 61, 1303-1313. [PubMed: 1694723]

(12). Miyake K, Underhill CB, Lesley J, and Kincade PW (1990) Hyaluronate Can Function as a CellAdhesion Molecule and Cd44 Participates in Hyaluronate Recognition. J. Exp. Med 172, 69-75. [PubMed: 2193100]

(13). Dreaden EC, Kong YW, Morton SW, Correa S, Choi KY, Shopsowitz KE, Renggli K, Drapkin R, Yaffe MB, and Hammond PT (2015) Tumor-Targeted Synergistic Blockade of MAPK and PI3K from a Layer-by-Layer Nanoparticle. Clin. Cancer Res 21, 4410-4419. [PubMed: 26034127]

(14). Dreaden EC, Morton SW, Shopsowitz KE, Choi JH, Deng ZJ, Cho NJ, and Hammond PT (2014) Bimodal Tumor-Targeting from Microenvironment Responsive Hyaluronan Layer-by-Layer (LbL) Nanoparticles. ACS Nano 8, 8374-8382. [PubMed: 25100313]

(15). Correa S, Boehnke N, Barberio AE, Deiss-Yehiely E, Shi A, Oberlton B, Smith SG, Zervantonakis I, Dreaden EC, and Hammond PT (2020) Tuning Nanoparticle Interactions with Ovarian Cancer through Layer-by-Layer Modification of Surface Chemistry. ACS Nano 14, 2224-2237. [PubMed: 31971772]

(16). Wilhelm S, Tavares AJ, Dai Q, Ohta S, Audet J, Dvorak HF, and Chan WCW (2016) Analysis of nanoparticle delivery to tumours. Nat. Rev. Mater. 1, 1-12.

(17). Sugahara KN, Teesalu T, Karmali PP, Kotamraju VR, Agemy L, Girard OM, Hanahan D, Mattrey RF, and Ruoslahti E (2009) Tissue-Penetrating Delivery of Compounds and Nanoparticles into Tumors. Cancer Cell 16, 510-520. [PubMed: 19962669]

(18). Laakkonen P, Porkka K, Hoffman JA, and Ruoslahti E (2002) A tumor-homing peptide with a targeting specificity related to lymphatic vessels. Nat. Med 8, 751-755. [PubMed: 12053175]

(19). Fogal V, Zhang L, Krajewski S, and Ruoslahti E (2008) Mitochondrial/cell-surface protein p32/ $\mathrm{gC1qR}$ as a molecular target in tumor cells and tumor stroma. Cancer Res 68, 7210-7218. [PubMed: 18757437]

(20). Pang HB, Braun GB, Friman T, Aza-Blanc P, Ruidiaz ME, Sugahara KN, Teesalu T, and Ruoslahti E (2014) An endocytosis pathway initiated through neuropilin-1 and regulated by nutrient availability. Nat. Commun 5, 1-12.

(21). Kwon EJ, Dudani JS, and Bhatia SN (2017) Ultrasensitive tumour-penetrating nanosensors of protease activity. Nat. Biomed. Eng 1, 1-10.

(22). Engelberth SA, Hempel N, and Bergkvist M (2014) Development of Nanoscale Approaches for Ovarian Cancer Therapeutics and Diagnostics. Crit. Rev. Oncog 19, 281-315. [PubMed: 25271436]

(23). Boehnke N, Correa S, Hao L, Wang W, Straehla JP, Bhatia SN, and Hammond PT (2020) Theranostic Layer-by-Layer Nanoparticles for Simultaneous Tumor Detection and Gene Silencing. Angew. Chem., Int. Ed 59, 2776-2783.

(24). Correa S, Boehnke N, Deiss-Yehiely E, and Hammond PT (2019) Solution Conditions Tune and Optimize Loading of Therapeutic Polyelectrolytes into Layer-by-Layer Functionalized Liposomes. ACS Nano 13, 5623-5634. [PubMed: 30986034] 
(25). Correa S, Choi KY, Dreaden EC, Renggli K, Shi A, Gu L, Shopsowitz KE, Quadir MA, BenAkiva E, and Hammond PT (2016) Highly Scalable, Closed-Loop Synthesis of Drug-Loaded, Layer-by-Layer Nanoparticles. Adv. Funct. Mater 26, 991-1003. [PubMed: 27134622]

(26). Kumar A, and Dixit CK Methods for characterization of nanoparticles Advances in Nanomedicine for the Delivery of Therapeutic Nucleic Acids; Elsevier Science, 2017; pp 43-58.

(27). Winther JR, and Thorpe C (2014) Quantification of thiols and disulfides. Biochim. Biophys. Acta, Gen. Subj 1840, 838-846.

(28). Fael H, and Demirel AL (2020) Nisin/polyanion layer-by-layer films exhibiting different mechanisms in antimicrobial efficacy. RSC Adv 10, 10329-10337.

(29). Mogaki R, Hashim PK, Okuro K, and Aida T (2017) Guanidinium-based "molecular glues" for modulation of biomolecular functions. Chem. Soc. Rev 46, 6480-6491. [PubMed: 29034942]

(30). Hunt H, Simon-Gracia L, Tobi A, Kotamraju VR, Sharma S, Nigul M, Sugahara KN, Ruoslahti E, and Teesalu $\mathrm{T}$ (2017) Targeting of $\mathrm{p} 32$ in peritoneal carcinomatosis with intraperitoneal linTT1 peptide-guided pro-apoptotic nanoparticles. J. Controlled Release 260, 142-153.

(31). Vinogradov AA, Yin YZ, and Suga H (2019) Macrocyclic Peptides as Drug Candidates: Recent Progress and Remaining Challenges. J. Am. Chem. Soc 141, 4167-4181. [PubMed: 30768253]

(32). Roth L, Agemy L, Kotamraju VR, Braun G, Teesalu T, Sugahara KN, Hamzah J, and Ruoslahti E (2012) Transtumoral targeting enabled by a novel neuropilin-binding peptide. Oncogene 31, 3754-3763. [PubMed: 22179825]

(33). Bradshaw JP (2003) Cationic antimicrobial peptides - Issues for potential clinical use. BioDrugs 17, 233-240. [PubMed: 12899640] 
A

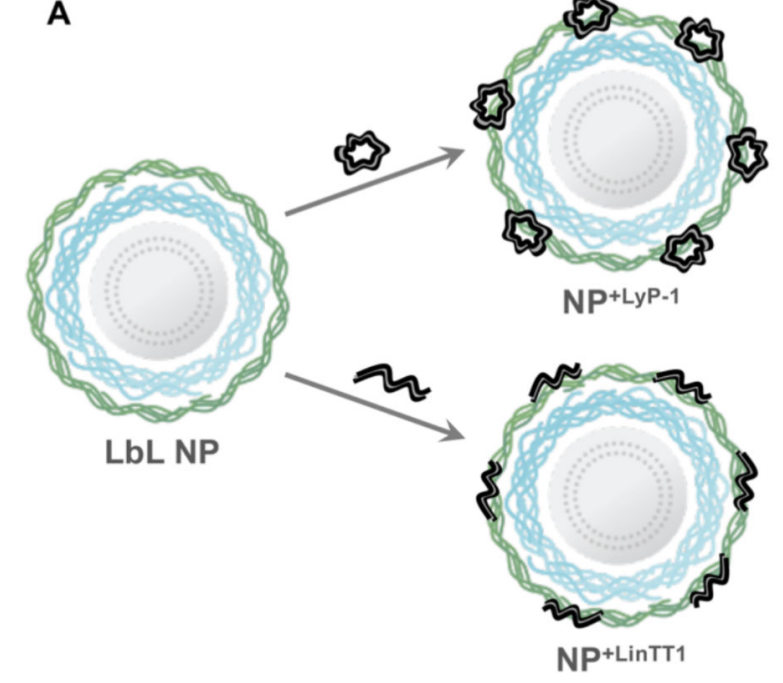

B

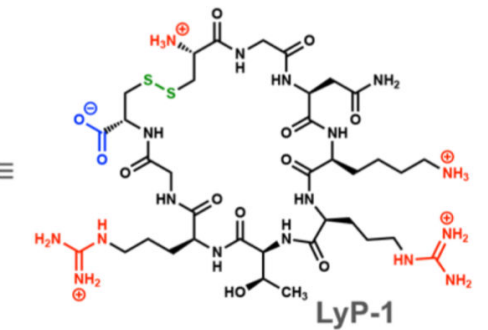

C

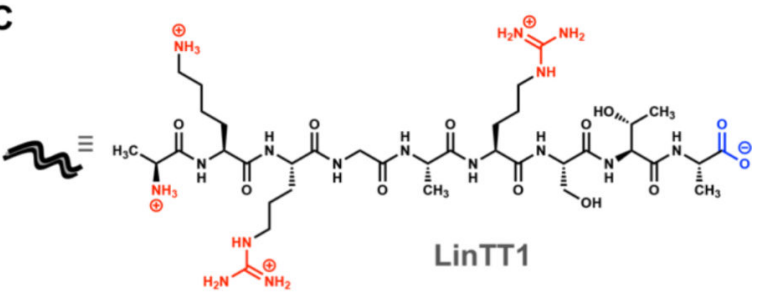

Figure 1.

(A) Electrostatic adsorption of cationic peptides enables surface functionalization of anionic LbL NPs. Structures of (B) LyP-1 and (C) LinTT1, with cationic residues indicated in red, anionic residues indicated in blue, and the disulfide bond indicated in green. 


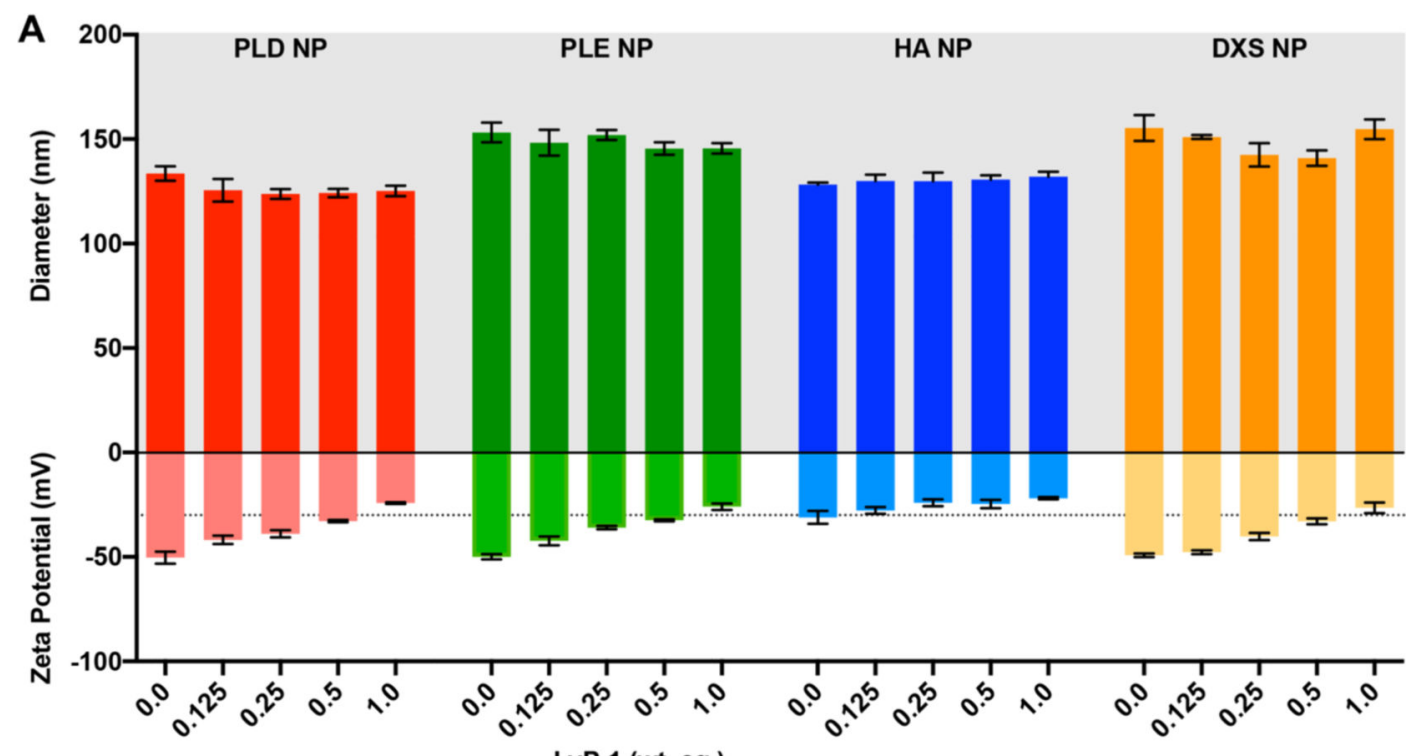

LyP-1 (wt. eq.)

B

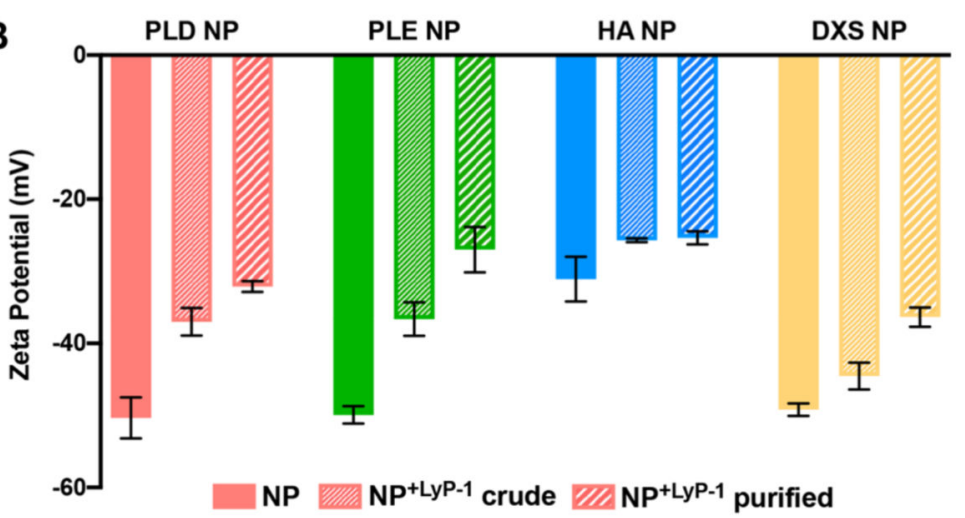

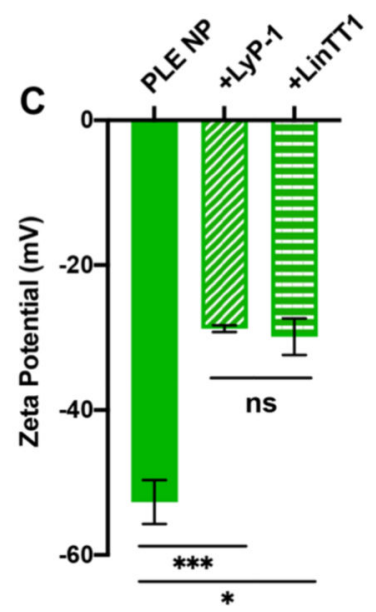

Figure 2.

(A) Effects of LyP-1 titration in $1 \mathrm{mM}$ HEPES onto the LbL NP library on particle size and zeta potential were monitored using dynamic light scattering. The $-30 \mathrm{mV}$ charge threshold for colloidal stability is indicated by a dashed line. (B) The zeta potential was measured before and after adsorption of $0.25 \mathrm{wt}$ equiv of LyP-1 onto the LbL NP library in $1 \mathrm{mM}$ HEPES as well as after purification of the modified NPs via tangential flow filtration. (C) Zeta potential measurements of PLE NPs after LyP-1 and LinTT1 adsorption (0.2 wt equiv) in $1 \mathrm{mM}$ HEPES and purification to remove unadsorbed peptide indicate similar interactions between the two peptides and the NP. Error bars represent the standard deviation of three measurements. $* p<0.05, * * * p<0.005$, determined using Student's $t$ test. 
A

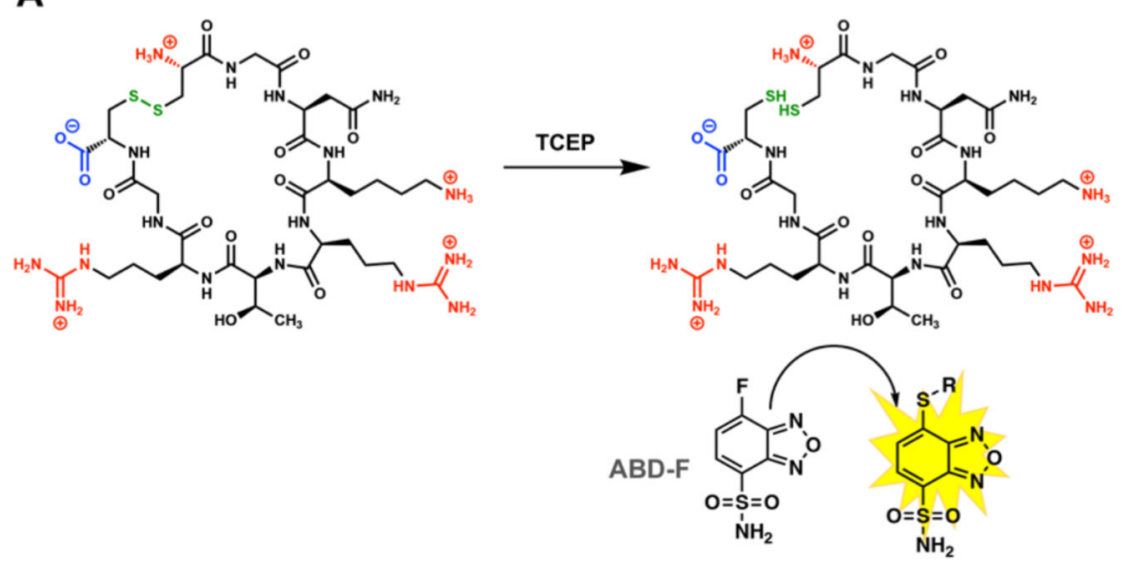

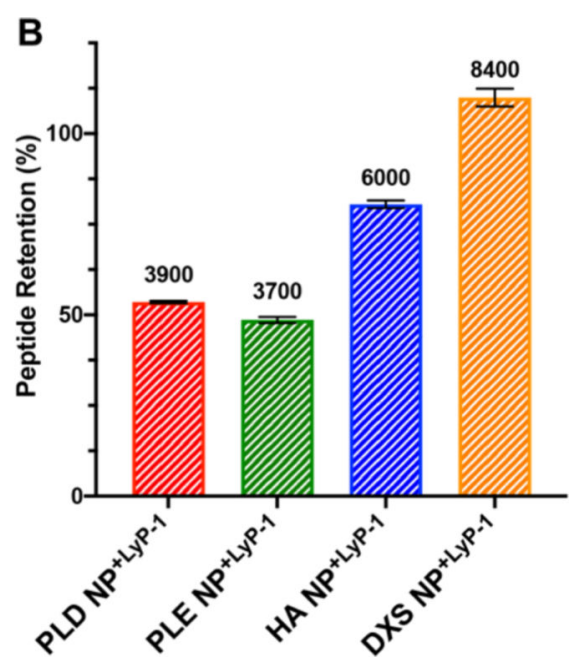

Figure 3.

(A) ABD-F assay scheme depicting LyP-1 reduction with TCEP and subsequent detection of free thiols with ABD-F, resulting in a fluorescent signal. (B) Quantification of LyP-1 retained on NPs after purification. Peptide retention was calculated as the post-TFF LyP-1 concentration divided by the pre-TFF concentration ( 0.2 wt equiv). The number of LyP-1 molecules per particle is indicated above each bar. Error bars represent standard deviation of three independent repeats. 

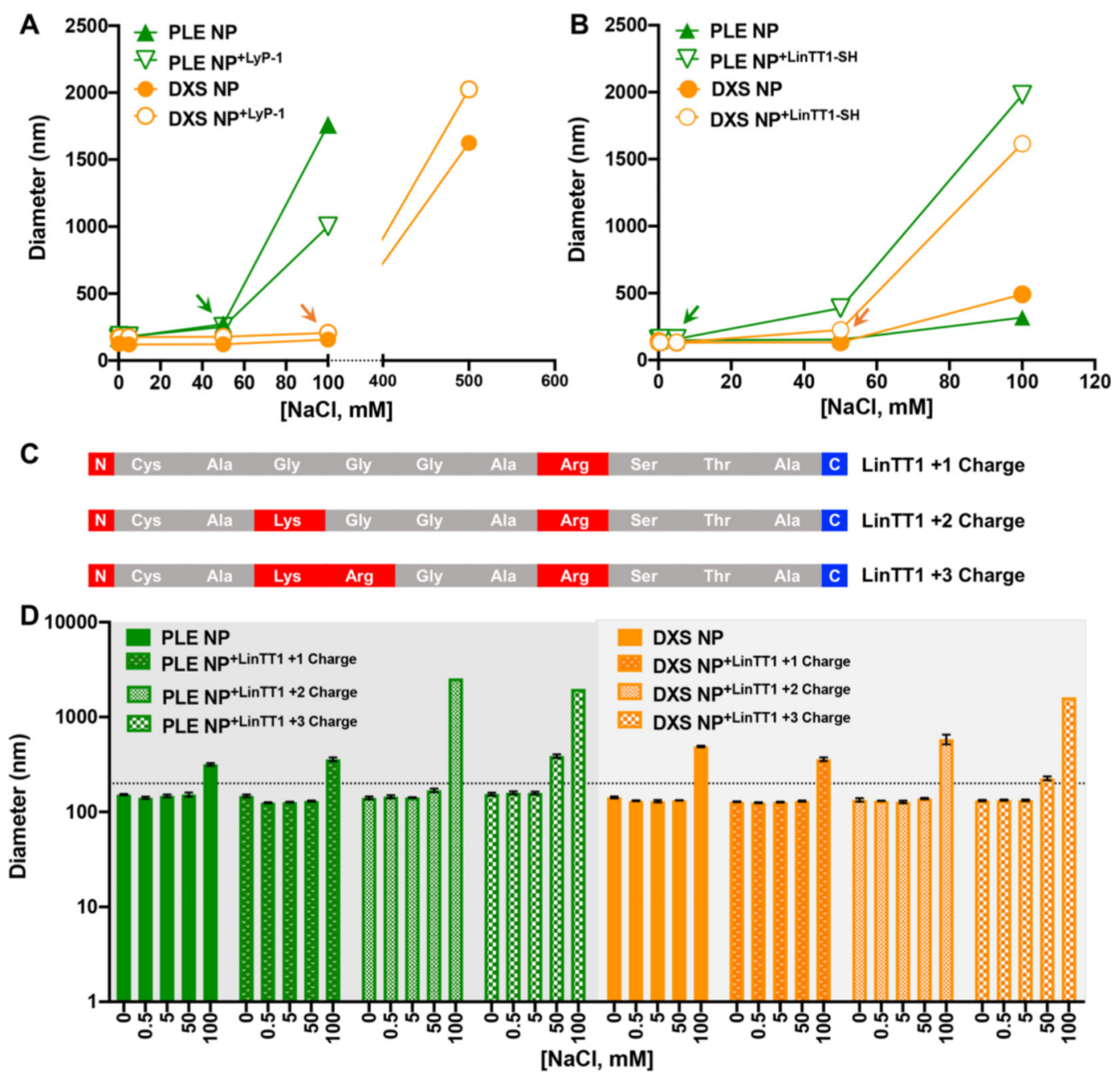

Figure 4.

Stability of PLE NPs and DXS NPs adsorbed with 0.5 wt equiv of (A) LyP-1 and (B) LinTT1 to increasing concentrations of $\mathrm{NaCl}$ as determined via DLS. Arrows denote thresholds for particle stability. (C) Peptide sequences of LinTT1 analogues are shown. Residues are colored red to denote cationic charge and blue to denote anionic charge. (D) Effect of peptide charge on stability of PLE NPs and DXS NPs adsorbed with LinTT1 analogues ( $0.5 \mathrm{wt}$ equiv) to increasing concentrations of $\mathrm{NaCl}$. A line placed at $200 \mathrm{~nm}$ denotes the threshold for NPs of stable size. Error bars represent the standard deviation of three repeat measurements. In panels A and B the error bars are smaller than the graph symbols and therefore omitted. Adsorption of the peptides tested in this figure was carried out in $25 \mathrm{mM}$ HEPES + $20 \mathrm{mM} \mathrm{NaCl}$. 


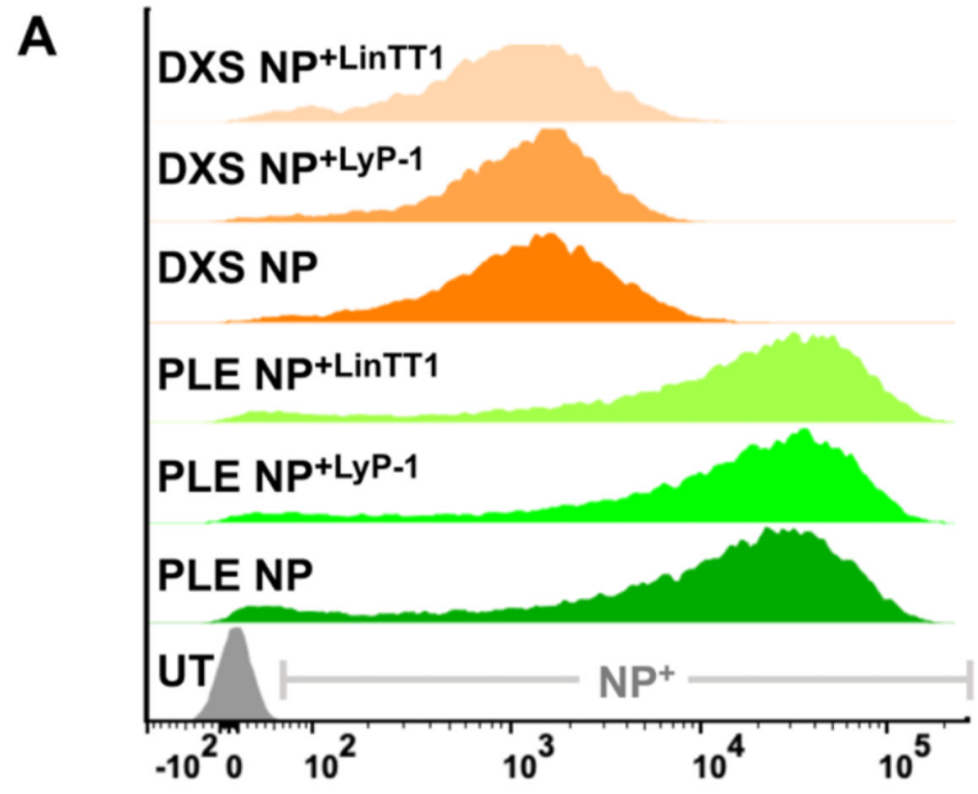

B

APC-A

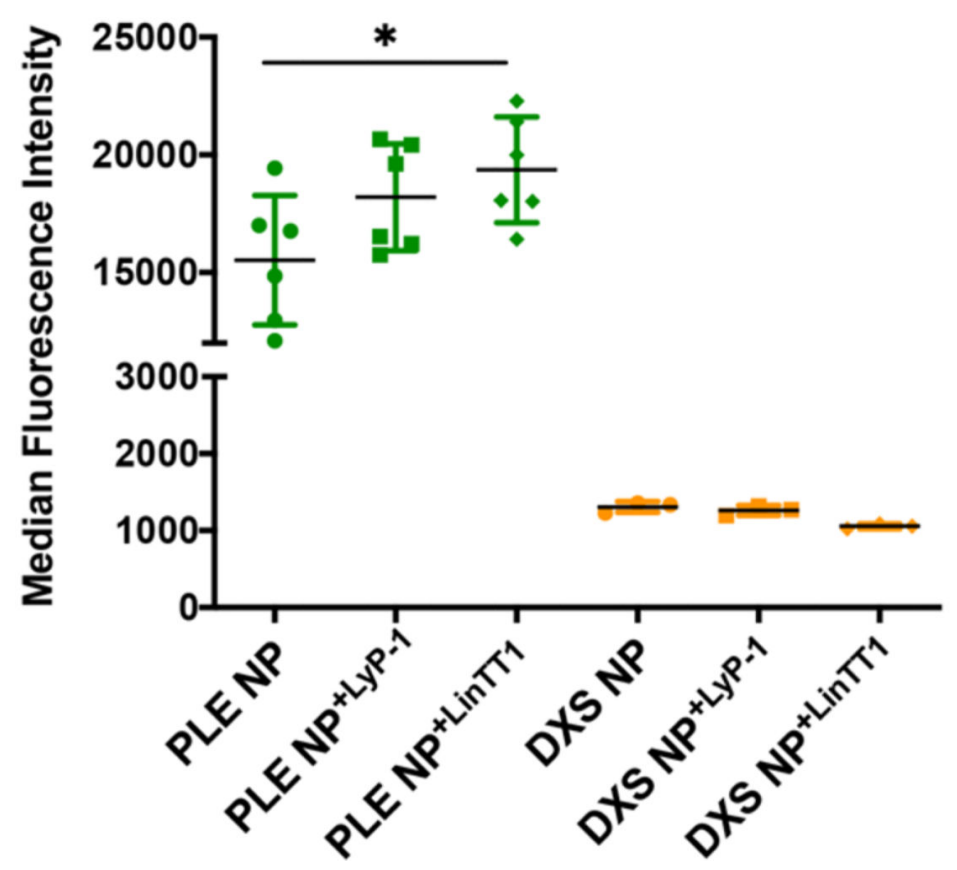

Figure 5.

(A) Flow cytometry was used to assess NP-cell association of 0.2 wt equiv of LyP-1 and LinTT1 functionalized LbL NPs with OVCAR8 cells at $24 \mathrm{~h}$, also represented as (B) median fluorescence intensity. For these experiments, peptides were adsorbed in water. ${ }^{*} p<0.05$, as determined using the Wilcoxon rank-sum test. 

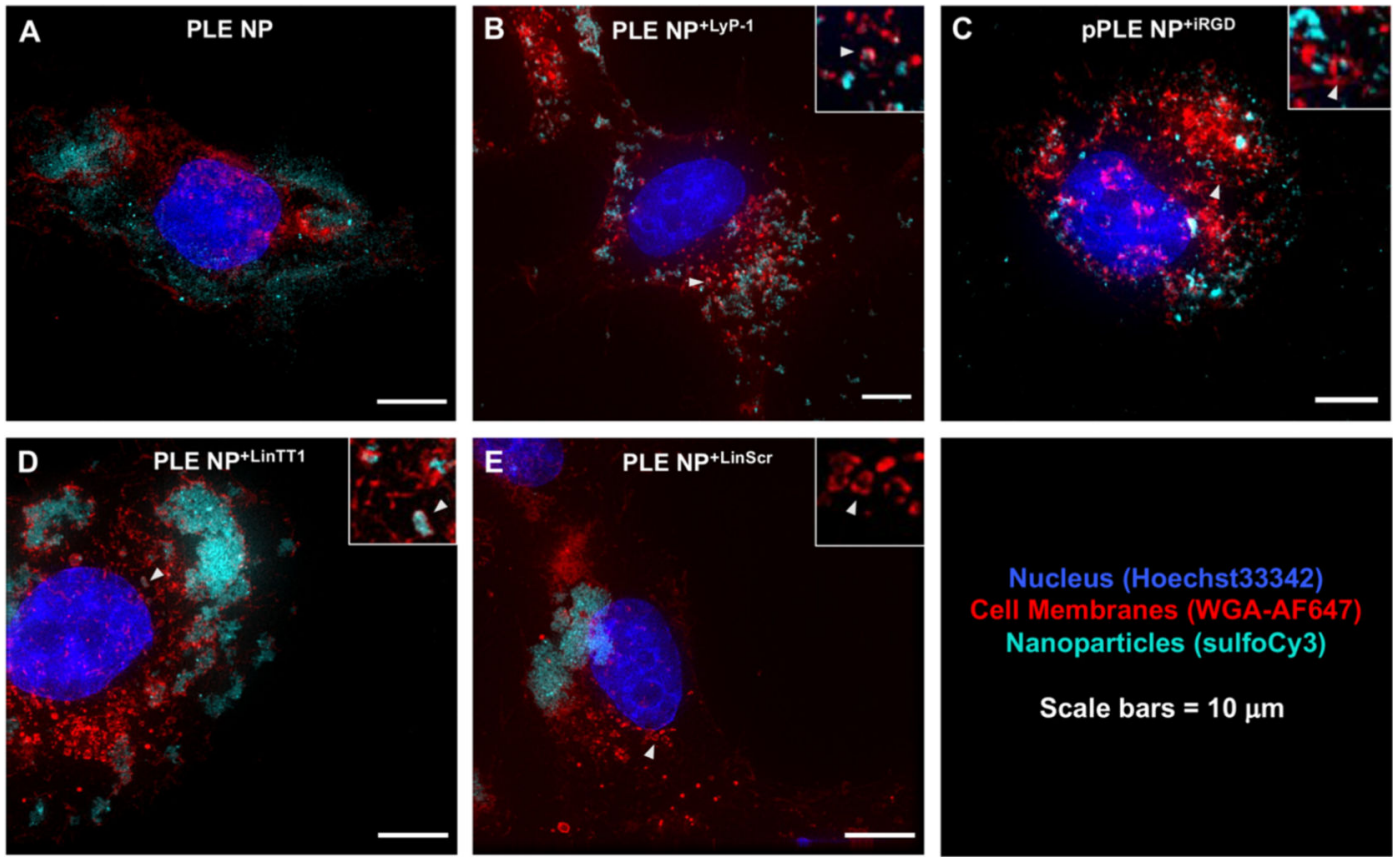

Figure 6.

Deconvolution microscopy analysis of OVCAR8 cells treated for $24 \mathrm{~h}$ with (A) PLE NPs, (B) PLE NPs ${ }^{+L y P-1}$ (C), pPLE LbL NPs ${ }^{+i R G D}$, (D) PLE NPs ${ }^{+L i n T T 1}$, and (E) PLE NPs +ScrTT1. Images are pseudocolored with red to represent membranes (wheat germ agglutininAF647), cyan to represent nanoparticles (sulfoCy3), and blue for the nuclei (Hoechst 33342). Scale bars $=10 \mu \mathrm{m}$. $0.3 \mathrm{wt}$ equiv of each peptide was adsorbed, with the exception of panel $\mathrm{C}$, in which case an equivalent amount of iRGD was utilized. Peptide adsorption was carried out in $25 \mathrm{mM}$ HEPES $+20 \mathrm{mM} \mathrm{NaCl}$. 